\title{
ON THE COMPACTIFICATION OF STRONGLY PSEUDOCONVEX SURFACES
}

\author{
VO VAN TAN ${ }^{1}$
}

\begin{abstract}
In this paper, we shall prove that the compactification of a strongly pseudoconvex surface is either a projective algebraic or an Inoue surface.

Furthermore, we shall construct an example of a strongly pseudoconvex surface $X$ which admits two distinct compactifications: One $M^{\prime}$ projective algebraic and the other one $M$ (highly) nonalgebraic.
\end{abstract}

Throughout this paper, $\mathbf{C}$-analytic surfaces (compact or noncompact) will mean 2-dimensional C-analytic manifolds. Purely 1-dimensional C-analytic spaces will be referred to simply as analytic curves.

1. The main problem.

Definition 1 [1]. A C-analytic surface $X$ is called strongly pseudoconvex if there exist

(i) a compact analytic curve $E \subset X$,

(ii) an exhaustion function $\phi \in C_{\mathbf{R}}^{\infty}(X)$ such that the Levi form

$$
L(\phi)_{x}:=\sum \frac{\partial^{2} \phi(x)}{\partial z_{i} \partial \bar{z}_{j}} d z_{i} d \bar{z}_{j}
$$

is positive definite for any $x \in X \backslash E$, with $1<i, j<2$.

REMARK. It can be proved that $E$ is actually exceptional in the sense of Grauert [1].

Definition 2 [4]. Let $M$ be a $C$-analytic surface and let $E \subset X$ be a compact analytic curve.

(a) $E$ is called an exceptional curve of the first kind if $E \stackrel{\sim}{\rightarrow} \mathbf{P}_{1}$ and $E^{2}=-1$.

(b) The $\mathbf{C}$-analytic surface $M$ is said to be minimal if $M$ is free from exceptional curves of the first kind.

From now on, all $\mathbf{C}$-analytic surfaces (compact or noncompact) are assumed to be minimal.

Let $M$ be a compact C-analytic surface and let $a(M):=$ the transcendental degree of the field of meromorphic functions on $M$. It is well known that $0 \leqslant a(M) \leqslant 2$. Following Kodaira [4, Vol. III], compact C-analytic surfaces can be classified as follows (see also [3]):

Received by the editors December 27, 1979 and, in revised form, September 12, 1980.

1980 Mathematics Subject Classification. Primary 32J05, 32J15; Secondary 32E05, 32F10.

Key words and phrases. Classification of compact C-analytic surfaces, exceptional curves of the first kind, minimal surfaces, Inoue surfaces, ruled surfaces, strongly pseudoconvex surfaces.

1 The author would like to thank the referee for his suggestions which led to the improvement of the presentation of this paper. 
THEOREM 1. Let $M$ be a compact $\mathrm{C}$-analytic surface. Then

(i) $M$ is projective algebraic iff $a(M)=2$.

(ii) $M$ is elliptic iff $a(M)=1$.

If $a(M)=0$, then $M$ must be one of the following types:

(iii) $M$ is a 2-dimensional torus.

(iv) $M$ is a $K_{3}$ surface i.e. $c_{1}(M)=b_{1}(M)=0$.

(v) $M$ is a nonelliptic Hopf surface i.e. $b_{2}(M)=0, b_{1}(M)=1$ and $M$ contains at least one analytic compact curve.

(vi) $b_{2}(M)=0, b_{1}(M)=1$ and $M$ contains no analytic curves.

(vii) $b_{2}(M) \geqslant b_{1}(M)=1$.

REMARKs. (a) Here $c_{1}(M)$ (resp. $b_{1}(M)$, resp. $b_{2}(M)$ ) denote the first Chern class (resp. the first Betti number, resp. the second Betti number) of $M$.

(b) Although a complete classification of compact $\mathbf{C}$-analytic surfaces of types (vi) and (vii) is not known yet, recently Inoue has constructed explicit examples of such surfaces [3].

Our main purpose here is to investigate the following

Problem A. Let $M$ be a compact $\mathbf{C}$-analytic surface and let us assume that there exists a compact analytic curve $\Gamma \subset M$ such that $X:=M \backslash \Gamma$ is strongly pseudoconvex. What are the analytic structures $M$ might be equipped with?

In the special case where $X$ is assumed to be Stein, this problem has been settled by Howard [2]. Our approach here is influenced by the technique in [2] and by recent construction of new compact $\mathbf{C}$-analytic surfaces without meromorphic functions [3].

REMARK. Since $X$ is strongly pseudoconvex, hence holomorphically convex, one can easily check that $\Gamma$ is connected. Let $\Gamma=\cup_{i} \Gamma_{i}$ where $\Gamma_{i}$ are the irreducible compact analytic curves.

2. The main theorem. We are now in a position to provide a complete answer to Problem A.

TheOREM B. Let $M$ be a compact $\mathrm{C}$-analytic surface and assume that there exists a compact analytic curve $\Gamma \subset M$ such that $M \backslash \Gamma=: X$ is strongly pseudoconvex admitting some exceptional curve $E$. Then either (i) $M$ is projective algebraic or (ii) $b_{2}(M)>b_{1}(M)=1$.

First of all we shall need the following result:

LEMma 2. Let $M, \Gamma, X$ and $E$ be as in Theorem B. Then the natural map

$$
r: H^{1}(M, C) \rightarrow H^{1}(\Gamma, \mathrm{C})
$$

is injective.

Proof. In fact one has the following exact sequence of cohomology groups with compact support:

$$
\cdots \rightarrow H_{c}^{1}(M \backslash \Gamma, \mathrm{C}) \rightarrow H^{1}(M, \mathrm{C}) \rightarrow H^{1}(\Gamma, \mathrm{C}) \rightarrow
$$


Since $M$ is nonsingular of R-dimension $=4$, Poincaré duality tells us that

$$
H_{c}^{1}(M \backslash \Gamma, \mathrm{C}) \simeq H_{3}(M \backslash \Gamma, \mathrm{C})=H_{3}(X, \mathrm{C}) .
$$

Since $X$ is strongly pseudoconvex, it follows from [5] that

$$
H_{3}(X, C) \simeq H_{3}(E, C)=0 .
$$

Hence it follows that $r$ is injective. Q.E.D.

Proof of Theorem B. Step 1. Let us assume that $a(M)=1$.

Following Kodaira [4] there exist a compact analytic curve $\Xi$ and an analytic map $\pi: M \rightarrow \Xi$ such that generically the fibres of $\pi$ are elliptic curves. Since $a(M)=1$, for any compact connected analytic curve $C \subset M, \pi(C)$ must be a point in $\Xi$ (see [4, Theorem 4.3, p. 1184]). Now since $E$ and $\Gamma$ are compact analytic curves in $M$, there exists a finite set $T \subset \Xi$ such that $\pi(E) \cup \pi(\Gamma) \subset T$. Hence for any $z \in \Xi \backslash T$, the elliptic curve $\Delta:=\bar{\pi}^{1}(z) \subset M \backslash(E \cup \Gamma) \simeq X \backslash E$. But the existence of the compact analytic subvariety $\Delta$ of positive dimension contradicts the strong plurisubharmonicity of $\phi$ on $X \backslash E$ (see Definition 1(ii)).

Step 2. Let us assume that $a(M)=0$.

(a) If $M$ is a 2-dimensional torus, an argument in [2] shows that $\Gamma$ must be a nonsingular elliptic curve. Consequently, $b_{1}(M)=4$ and $b_{1}(\Gamma)=2$. But this will contradict the injectivity of the map $r$ in Lemma 2.

(b) If $M$ is a $K_{3}$ surface, let $L$ be the line bundle determined by the divisor $\Gamma=\cup \Gamma_{i}$ and let $a_{i j}:=\Gamma_{i} \cdot \Gamma_{j}$.

In view of the hypothesis that $a(M)=0$, an argument in [2] and [4] shows that the matrix $\left(a_{i j}\right)$ must be negative definite.

Claim. $M$ cannot be the compactification of any strongly pseudoconvex surface.

In fact if it were, in view of the negative definiteness of $\left(a_{i j}\right)$, a result in [3] tells us that there exist a 2-dimensional normal compact $\mathbf{C}$-analytic space $\hat{M}$, a point $\{*\} \subset \hat{M}$ and a surjective holomorphic map $\boldsymbol{\theta}: \boldsymbol{M} \rightarrow \hat{\boldsymbol{M}}$ inducing a biholomorphism $M \backslash \Gamma \simeq \hat{M} \backslash\{*\}$. Since $\hat{M} \backslash\{*\} \simeq X$ is holomorphically convex, in view of Riemann's extension theorem, any global holomorphic function on $\hat{M} \backslash\{*\}$ can be extended to $\hat{M}$. This implies the existence of nonconstant global holomorphic functions on the compact analytic space $\hat{M}$. Contradiction!

Step 3. Let us consider the following natural map

$$
\begin{aligned}
\alpha: H^{2}(M, \mathbf{C}) & \rightarrow H^{2}(E, \mathbf{C}) \\
c_{1}(E) & \mapsto E \cdot E .
\end{aligned}
$$

Since $E$ is exceptional, $E^{2}<0$. Therefore $\alpha$ is not a zero map. Consequently $H^{2}(M, \mathrm{C})$ and hence $b_{2}(M) \neq 0$.

In view of Theorem 1 above, $M$ is either projective algebraic or $b_{2}(M)>b_{1}(M)$ = 1. Q.E.D.

3. The main example. We are now in a position to exhibit an example showing that both alternatives (i) and (ii) in Theorem B indeed occur (and even simultaneously). 
EXAMPle C. In [3] a compact C-analytic surface $M$ is explicitly constructed with $b_{1}(M)=1$. Furthermore, $M$ contains exactly two compact analytic curves:

(i) a rational curve $\Gamma$ with an ordinary double point with $\Gamma^{2}=0$,

(ii) an elliptic curve $E$ with $E^{2}=-1$.

Also it is proved that $X:=M \backslash \Gamma$ is biholomorphic to a holomorphic line bundle over $E$ with $E$ as its zero section. In view of (ii) a result in [1] tells us that $X$ is actually a strongly pseudoconvex manifold admitting $E$ as its exceptional curve. Moreover, since $K=[-\Gamma-E]$ where $K$ is the canonical line bundle on $M$, one has, in view of (i) and (ii), $b_{2}(M)=-K^{2}=1$.

On the other hand since $X$ is biholomorphic to a line bundle, the fibres of the latter can be projectivized so that $X$ can be realized as a Zariski open subset of some ruled surface, say $M^{\prime}$ over $E$.

Consequently, we obtain here an example of a strongly pseudoconvex surface which admits 2 distinct compactifications: One projective algebraic $M^{\prime}$ and the other one $M$, (highly) nonalgebraic.

\section{REFERENCES}

1. H. Grauert, Über Modifikationen und exzeptionelle analytische Mengen, Math. Ann. 146 (1962), 331-368.

2. A. Howard, On the compactification of Stein surfaces, Math. Ann. 176 (1968), 221-224.

3. M. Inoue, New surfaces with no meromorphic functions, Proc. Internat. Congr. Math., Vancouver, Vol. I, 1974, pp. 423-426.

4. K. Kodaira, Collected works. Vols. I, III, Princeton Univ. Press, Princeton, N. J., 1975.

5. Vo Van Tan, On the classification of q-convex complex spaces by their compact analytic subvarieties, Ph.D. Thesis, Brandeis University, Waltham, Mass., 1974.

Department of Mathematics, Tufts University, Medford, Massachusetts 02155 